\title{
Esophageal cancer: challenges, concerns, and recommendations for management amidst the COVID-19 pandemic
}

\section{Tania Triantafyllou, ${ }^{\text {, Michael T. Olson }}{ }^{\mathrm{b}}$, Dimitrios Theodorou a, Georgios Zografos ${ }^{\mathrm{a}}$, Saurabh Singhalc}

Hippocration General Hospital of Athens, National and Kapodistrian University of Athens, Athens, Greece; University of Arizona College of Medicine - Phoenix Campus, Phoenix, AZ, USA; Indraprastha Apollo Hospital, New Delhi, India

Abstract

\begin{abstract}
Since December 2019, the outbreak of coronavirus disease 2019 (COVID-19) has rapidly spread worldwide, raising great concern, particularly in immunosuppressed cancer patients. The pandemic situation remains extremely dynamic, which necessitates proactive management decisions from oncologists and oncologic surgeons in effort to mitigate the risk of both SARS-CoV-2 infection and cancer metastasis. Esophageal cancer, in particular, is one of the deadliest types of malignancy worldwide, reflecting both aggressive biology and a lack of adequate treatment. Several challenges and concerns regarding the management of esophageal cancer have been raised in light of the ongoing viral pandemic. The primary aim of this review is to summarize the salient evidence for recommendations and optimal treatment strategies for patients with esophageal cancer amidst the COVID-19 pandemic.
\end{abstract}

Keywords COVID-19, coronavirus, SARS-CoV-2, esophageal cancer, esophagectomy

Ann Gastroenterol 2020; 33 (4): 1-6

\section{Introduction}

An acute respiratory disease caused by a novel coronavirus (SARS-CoV-2), coronavirus disease 2019 (COVID-19) has gripped the world in a pandemic and challenged global culture, economy and healthcare infrastructure. The outbreak of COVID-19 initially developed in the city of Wuhan, Hubei, province of China, in early December 2019 [1]. Molecular techniques and unbiased DNA sequencing led to the discovery of SARS-CoV-2, indicating a novel betacoronavirus belonging to the Sarbecovirus subgenus of the Coronaviridae family [1]. According to the most recently reported data by international

aDepartment of Surgery, Hippocration General Hospital of Athens, National and Kapodistrian University of Athens, Athens, Greece (Tania Triantafyllou, Dimitrios Theodorou, Georgios Zografos); ${ }^{\text {bDepartment }}$ of Surgery, University of Arizona College of Medicine - Phoenix Campus, Phoenix, AZ, USA (Michael T. Olson); 'Department of GI Surgery and Liver Transplantation, Indraprastha Apollo Hospital, New Delhi, India (Saurabh Singhal)

Conflict of Interest: None

Correspondence to: Dr. Tania Triantafyllou, MD, Department of Surgery, Hippocration General Hospital of Athens, National and Kapodistrian University of Athens, Athens, Greece, Vas. Sofias Avenue 114, 11527 Athens, Greece, e-mail: t_triantafilou@yahoo.com

Received 2 April 2020; accepted 17 May 2020; published online 22 June 2020

DOI: https://doi.org/10.20524/aog.2020.0519 authorities on June 1, 2020, COVID-19 has been confirmed in more than 6 million cases globally, spreading at a rapid rate, with little indication of slowing despite best efforts to mitigate transmission. This seventh member of the family of coronaviruses has presently resulted in the deaths of more than 370,000 patients worldwide. The prodromal symptoms are non-specific and mainly include fever, dry cough and malaise, while the advanced clinical syndrome is characterized by severe pneumonia [2]. Its clinicopathologic characteristics mimic the other well-known coronavirus strains, SARS-CoV originating from China, and MERS-CoV from Saudi Arabia, both related to a fatal illness $[3,4]$. Beyond evidence-based supportive care, the cornerstone in the management of severe respiratory viral infections, no coronavirus antiviral medications or vaccines of proven efficacy have been established for treatment [5]. COVID-19 continues to raise considerable challenges worldwide, and it has been pronounced a Public Health Emergency of International Concern by the World Health Organization (WHO).

It has become increasingly important for healthcare systems and clinicians to adopt a universal, consolidated approach to patient care amidst the ongoing viral pandemic, particularly for vulnerable populations. Cancer patients, and more precisely, immunocompromised patients, may be susceptible to severe illness with potentially fatal consequences [6]. However, existing data on the association between cancer and COVID-19 remain insufficient because of the small numbers of cancer patients that have been investigated, mainly in Eastern countries. The true burden on patients receiving chemo(radio)therapies and 
medical immunosuppression, as well as transplant recipients and select surgical patients, is yet to be quantified. A recent nationwide study from China revealed an increased risk of severe symptom presentation of SARS-CoV-2 infection among cancer patients [7]. Esophageal cancer (EC), in particular, is one of the deadliest types of malignancy worldwide, reflecting both aggressive biology and a lack of adequate treatment. Now, while oncologic and gastrointestinal surgeons face the outbreak of COVID-19, the optimal management of these patients may be even more controversial and problematic than before. Unique considerations may be necessary for: (a) patients newly diagnosed with EC without any previous treatment; (b) non-responding or partial-responding patients once re-staged after neoadjuvant therapy; (c) complete responding patients after neoadjuvant therapy; (d) patients after esophagectomy (hospitalized or not); and (e) those in need of palliative care. These subgroups should be provided with an individualized treatment strategy, not necessarily following the well-established approaches. Therefore, the primary aim of this review is to summarize the salient evidence for recommendations and optimal treatment strategies for patients with EC amidst the COVID-19 pandemic.

\section{New diagnosis of EC}

When EC is newly diagnosed, the decision for the most appropriate treatment strategy normally falls upon a multidisciplinary care team, who remain responsible for the patient. In a viral pandemic, clinicians should balance the need to perform life-prolonging surgery and offer perioperative therapies with a patient's risk of contracting severe pneumonia if infected with SARS-CoV-2. Treatment strategies may need to be based on the pathophysiology of 2 major variants, squamous cell carcinoma or adenocarcinoma, and recommendations may thus differ. Patients diagnosed with early EC cT1aN0M0 (UICC classification 7th edition), regardless of the histologic subtype, will benefit from an endoscopic resection with a sameday discharge if the procedure is uneventful, since endoscopic mucosal resection and endoscopic submucosal dissection are considered minimally invasive techniques [8]. Whether endoscopic therapy for this indication should be postponed, given the risk of progression to a more advanced stage during the pandemic, remains unclear. In patients with lesions limited to the submucosa (cT1b), an esophagectomy remains ideal. While most patients who get infected remain asymptomatic carriers, or develop only mid flu symptoms, the high morbidity and mortality in severe cases should dictate a highly cautious approach with a significant index of suspicion. It is highly imperative that an alternative "rescue" approach might be considered. Endoscopic resection with intense endoscopic imaging follow-up based on the risk of the involved lymph node disease (e.g., up to 50\%), radicality of resection (e.g., status of resection margins), and high-risk histologic features (e.g., tumor size, grade of differentiation) may be feasible, but should only be applied after individualized multidisciplinary decision making [9-11]. More precisely, high-risk early EC, including lesions with positive lymphovascular invasion, poor differentiation, ulcerative pattern of invasion or deeper than T1a, should be meticulously reevaluated and restaged in an effort to eliminate the risk of underestimated lymph node disease balancing the risk of undetected lymph node metastases. When remaining or recurrent disease cannot be excluded, interdisciplinary decisions for more radical interventions should remain the standard of care. Clinicians have asked what the chances of dissemination are during surveillance, considering the possible concurrent presence of positive lymph nodes leading to unresectable disease. Given the almost excellent results after radical surgery for early EC, the discussion is rather inconclusive. Another measure that may have relative success for high-risk, resected lesions could be additional chemoradiotherapy. Such practice has been implemented with acceptable results in previous observational studies for squamous cell carcinoma, mainly in the Eastern hemisphere for patients who were not surgical candidates [12]. However, this approach may be inadequate from an oncologic standpoint, especially regarding cT1bN0 lesions, which present a high risk for lymph node metastases. Endoscopic procedures, however, are to be undertaken with much caution, per the advisory of the Society of American Gastrointestinal and Endoscopic Surgeons, as any luminal or airway procedures pose a high risk of aerosol transmission.

\section{Elective procedures and preventive strategies}

Updated guidelines published by the WHO and the American College of Surgeons recommend delay of elective surgeries. Meanwhile, the American Society of Clinical Oncology has encouraged the delay of surgeries that would not otherwise result in detrimental harm to patients' health [13]. The National Health Service (NHS) in the United Kingdom recently classified surgery for cancer patients as priority level 2 following emergency and urgent operations (within 72 h) [14]. Priority level 2 requires "clean sites". Herein, the risk of potentiating metastatic disease (thus making the disease inoperable) should be balanced against the risk of contracting COVID-19 in the perioperative period. The treatment of more advanced stages of EC (>stage I) should not be delayed, given the considerable metastatic potential of this type of malignancy. Neoadjuvant therapy may be prioritized, given the improved results compared to surgery alone. Although surgery upfront is considered to be a valid option for EC stage II, according to Mariette et al [15], there is currently no strong evidence in the literature to suggest that neoadjuvant therapy should not be the first approach before radical surgical resection, as underlined by the Japanese and western guidelines for both adenocarcinoma and squamous cell carcinoma $[8,16,17]$. Those guidelines indicate that induction therapy is an acceptable option for patients diagnosed with stage II, who may benefit from withholding the surgical plan as scheduled after completion of neoadjuvant therapy and restaging. Beginning with chemo(radio)therapy based on the guidelines of each country requires up to 2 months for completion of 
the neoadjuvant setting and re-staging, a time period which may be life-saving until the pandemic comes to a plateau. To this end, the patient may receive a one-day chemotherapy regimen and be discharged. According to the Chinese Medical Association, 1-2 additional cycles of chemotherapy may be administered in patients who have completed preoperative therapy, but failed to undergo planned surgery $[18,19]$. Recently, an expert group in the United Kingdom formed an evidence-based consensus on guiding principles with regard to radiotherapy. Interestingly, the panel suggested that definite chemoradiation may be the most appropriate approach during the pandemic, mainly because of the uncertain availability of surgical procedures and bed capacity, while unfractionated definite radiotherapy of $50 \mathrm{~Gy} / 16$ fractions may selectively be acceptable as a second option [20]. Another multicenter review outlined the importance of careful estimation of the risk benefit for patients under adjuvant treatment. Overall, treatment strategies not directly related to improved overall survival could be postponed, according to the authors. Meanwhile, because these individuals are immunocompromised, clinicians should educate patients about the importance of performing selfprotective measures, such as frequent hand hygiene, avoidance of crowds and, most importantly, social distancing. All such hygiene strategies should be strictly applied during visits for laboratory testing surveillance, when needed. Home quarantine must be encouraged in these patients for an extended period, ranging from at least 14 days to as long as 4-6 weeks. Moreover, cancer-related cachexia and nutritional impairment are extremely frequent at the time of diagnosis and predispose to an increased risk of infection. We recommend that daily nutrition should be supported with supplements, in case of inadequate caloric intake, in order to optimize the performance status before surgery. According to the recommendations provided by the Chinese Medical Association, sufficient hydration and daily physical activity for at least 30 min could improve the physical condition before surgery [18]. Patients who are inherently immunocompromised, such as patients with inadequatelycontrolled diabetes, are at higher risk, and rigorous management strategies are required to prevent infection in these individuals.

\section{EC after neoadjuvant treatment}

An active surveillance strategy after the completion of neoadjuvant therapy has been evaluated in other types of cancer, with promising results [21]. Ongoing investigations are evaluating this strategy for EC, in both patients cured by neoadjuvant therapy alone and those with subclinical disseminated disease at the time of completion of neoadjuvant therapy [22]. Active surveillance strategies are presently applied in selected patients with EC who decline surgery, or in those deemed unfit for major surgical interventions upon completion of neoadjuvant therapy. The active surveillance strategy could be an alternative approach for clinically complete responders after neoadjuvant therapy in an effort to avoid possible exposure to COVID-19, which may be particularly beneficial considering the patients' immunosuppressed status [23]. Previous studies have demonstrated an improved pathological complete response after a prolonged time between completion of neoadjuvant therapy and esophagectomy among complete responders [24]. In fact, endoscopic and imaging reassessment of these patients may be scheduled 6-8 weeks after completion of induction therapy, and a non-surgical pathway could be intensively considered based on diagnostic modalities (e.g., bite-on-bite endoscopic biopsies, endoscopic ultrasound, positron emission tomography-computed tomography scanning) [25]. Similarly to the organ-preserving approach in rectal cancer patients who present a complete response after neoadjuvant or definite therapies, the "active surveillance" pathways have been designed as a rationale for clinical complete responders after neoadjuvant chemoradiotherapy in the treatment of EC. Currently, there are 2 ongoing randomized trials that aim to compare this organ-sparing approach to the standard of care (combined neoadjuvant therapy plus esophagectomy), the SANO trial and the Esostrate trial [26-28]. Recruitment is not yet complete and the results are still eagerly awaited. Meanwhile, the preSINO trial protocol, a collaboration between The Netherlands and a few Chinese centers, was recently published [29]. To date, there has only been one retrospective study from The Netherlands that presented an improved 3-year overall survival between patients under active surveillance after neoadjuvant chemoradiotherapy over individuals after neoadjuvant therapy plus surgery [23]. The main argument against the "surgery as needed" therapeutic approach is the possibility of misdiagnosed remaining or disseminated recurrent disease, despite the apparent clinical complete response after induction therapies. Thus, the active surveillance approach, although appealing during the pandemic (especially in countries where it is reaching its peak or areas presenting a "very high" plateau), should be only considered after multidisciplinary meetings and only for selected patients. Most importantly, older patients, or those who are not strong surgical candidates because of their comorbidities, might be in favor of a "wait-and-see" option during this challenging period. Informing the patient of the diagnosis and obtaining consent are significant and mandatory before any of the available therapeutic options are decided. Moreover, all recommendations regarding protective measures for patients and healthcare personnel during the follow-up endoscopic procedures should be strictly encountered [27]. With respect to the study by Liang et al [7], patients who underwent recent chemotherapy (or surgery) presented a higher risk of severe events when infected by COVID-19. Therefore, protective measures should be meticulously preserved after neoadjuvant therapies. Ultimately, decision-making in these cases can be quite controversial; delayed surgery with close monitoring for disease should be a shared decision between patient and physician at the conclusion of an informed discussion of the short- and long-term risks.

\section{Esophagectomy}

In spite of the ongoing and unpredictable outbreak, esophagectomy may be an inevitable approach to treating EC in select patients. Patients who did not respond, or 
only partially responded to neoadjuvant therapy for locally advanced EC, may lose their "curative window", depending on a radical resection, which may compromise survival. Those unwilling to take the risk of avoiding immediate surgery should be offered esophagectomy in referral centers under strict infection control measures. Minimally invasive esophagectomy should be performed with efforts to eliminate surgical trauma, shorten perioperative time and minimize operative blood loss. Enhanced recovery protocols after surgery have a well-documented benefit over the standard care protocol in EC surgery and should be incorporated in an effort to accelerate recovery and reduce hospital stay [30], aiming to decrease the risk of viral exposure. Depending on the region's health system instructions, in-hospital quarantine (with strict isolation from the outside world and only limited healthcare staff permitted in and around the patient's room, for a period of at least 2 weeks) with concurrent observation of body temperature (at least twice per day) may be considered. Obtaining routine laboratory tests (e.g., white blood cell count, C-reactive protein, procalcitonin, and rapid influenza diagnostic test) and chest X-ray or chest computed tomography scans would contribute to optimizing patient safety prior to surgery [18]. Experience from China disclosed that $1 \%$ of COVID-19-positive patients had a history of cancer, while cancer patients with COVID-19 presented an increased risk for severe disease [7]. Therefore, if proven positive before surgery, the patient should be supported conservatively, and surgical planning should be postponed until recovery. Postoperative isolation should be employed. Constant awareness and support from the anesthesiology and intensive care teams is mandatory. Finally, follow up of uneventfully discharged patients could be replaced by teleconferencing in contact with the therapeutic team of healthcare providers, including psychological support, if possible.

\section{Palliative care}

According to a few experts, patients with advanced disease, but without symptoms suggestive of SARS-CoV-2 infection, should ideally continue with their planned radiotherapy sessions to achieve symptom palliation [13,31]. Additionally, when palliative care has been administered, forced quarantine, as imposed in most countries worldwide, should be supported by the respective team of internists and primary care specialists responsible for pain control and feeding route maintenance $[32,33]$. On the other hand, other physicians state that palliative medical treatments at home should ideally replace radiation therapy until the disease reaches a plateau point, especially in high-risk areas such as in Italy. EC patients with unresectable, obstructive tumors might benefit from endoscopic stent placement in order to facilitate oral feeding. Most importantly, expert panels, medical ethicists and palliative support groups will play a vital role in facing these challenging issues given the pragmatic aspects of healthcare resources available during this ongoing viral pandemic (Table 1).
Table 1 A summary of treatment strategies for esophageal cancer during the coronavirus disease 2019 (COVID-19) pandemic

New diagnosis of esophageal cancer
Consider endoscopic therapy for new diagnosis of early stage
esophageal cancer (stage T1a/b superficial)
Perform surgery as soon as feasible in patients with esophageal
cancer T1b or greater
If eligible for adjuvant therapy, consider neoadjuvant therapy
Follow-up with imaging based on risk of involved lymph node
disease, radicality or resection, and high-risk histologic features
No response or partial response after neoadjuvant therapy
Begin chemo(radio)therapy based on the guidelines of the
respective country
Educate immunocompromised patients on the importance of self-
protective viral pandemic measures
Supplement daily nutrition in cases of inadequate caloric intake
Complete response after neoadjuvant therapy
Consider active surveillance strategy to mitigate risk of possible
exposure to COVID-19
Schedule endoscopic and imaging reassessment at 6-8 weeks after
completion of induction therapy
Implement non-surgical, intensive monitoring (e.g., bite-on-bite
endoscopic biopsies, endoscopic ultrasound, positron emission
tomography-computed tomography scanning)
Inform patient of risks of delayed surgery and close monitoring
approach
Esophagectomy
In non-responding or partial-responding patients to neoadjuvant
therapy for locally-advanced esophageal cancer, offer minimally-
invasive esophagectomy in referral center under strict infection
control measures
Utilize enhanced recovery after surgery protocol
secomments with advanced disease, without symptoms of COVID-19,
Forced quarantine should be supported by primary care
specialists responsible for pain control and feeding maintenance
Obtain routine laboratory tests (white blood cells, C-reactive
protein, procalcitonin, rapid influenza diagnostic test) with chest
x-ray or chest computed tomography
Replace routine postoperative follow-up visits with teleconference
sessions

Treatment strategies presented herein are based on limited data available to inform clinical and surgical decision-making for patients with esophageal cancer during the ongoing COVID-19 pandemic. Decisions may be altered based on COVID-19 burden at respective institutions, availability of hospital resources (intensive care unit beds, ventilators, clinicians, personal protective equipment), and the COVID-19 trajectory within the hospital

\section{Concluding remarks}

Healthcare officials and international organizations have extrapolated recommendations regarding clinical practice in support of patients and healthcare providers worldwide. Updated protocols are being published in an effort to guide physicians and multidisciplinary teams responsible for the treatment of the cancer patient during the COVID-19 pandemic. Comprehensive management of EC should emphasize carefully-designed, therapeutic strategies that may 
Table 2 Perioperative recommendations for esophageal cancer procedures during the coronavirus disease 2019 (COVID-19) pandemic

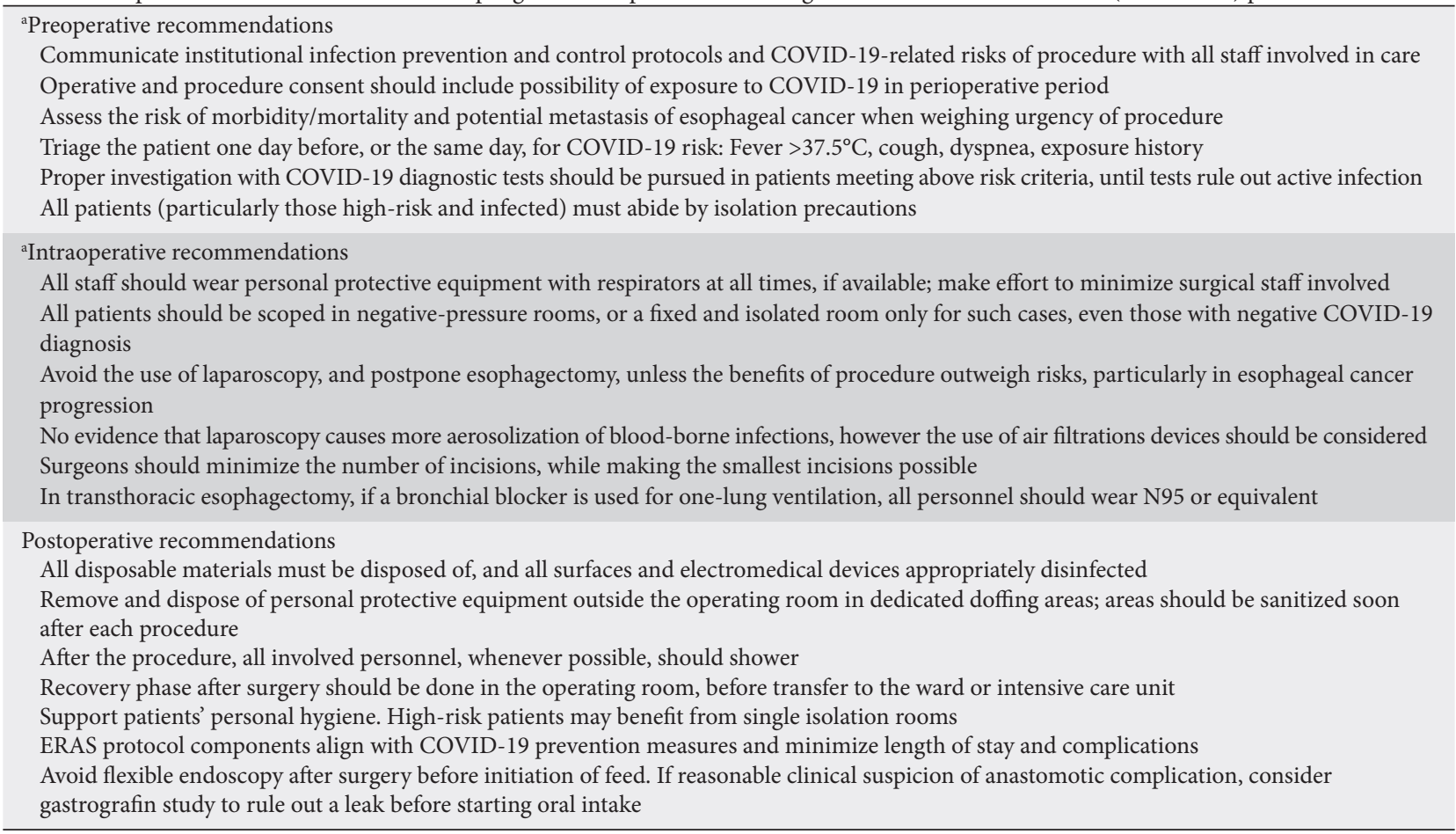

Recommendations presented herein are based on limited data available to inform appropriate perioperative management of patients with esophageal cancer necessitating procedural/surgical intervention

${ }^{a}$ Recommendations are adapted from ISDE Guidance Statement: Management of upper-GI Endoscopy and Surgery in COVID-19 outbreak (2020 Mar 31). Course of action may be altered based on COVID-19 burden at respective institutions, availability of hospital resources (intensive care unit beds, ventilators, clinicians, personal protective equipment), and the COVID-19 trajectory within the hospital

be tailored to the patient, taking under consideration the aggressive nature of the malignancy, as well as the evolving limitations and concerns of the healthcare infrastructure. These recommendations for managing the care of EC patients are likely to be adapted according to the rapidly changing circumstances of the ongoing pandemic (Table 2).

\section{References}

1. Zhu N, Zhang D, Wang W, et al; China Novel Coronavirus Investigating and Research Team. A novel coronavirus from patients with pneumonia in China, 2019. N Engl J Med 2020;382:727-733.

2. Chan JF-W, Yuan S, Kok K-H, et al. A familial cluster of pneumonia associated with the 2019 novel coronavirus indicating personto-person transmission: a study of a family cluster. Lancet 2020;395:514-523.

3. Su S, Wong G, Shi W, et al. Epidemiology, genetic recombination, and pathogenesis of coronaviruses. Trends Microbiol 2016;24:490-502.

4. Wang Z, Yang B, Li Q, Wen L, Zhang R. Clinical features of 69 cases with coronavirus disease 2019 in Wuhan, China. Clin Infect Dis 2020 Mar 16 [Epub ahead of print]. doi: 10.1093/cid/ciaa272

5. Clinical management of severe acute respiratory infection when novel coronavirus (2019-nCoV) infection is suspected: Interim guidance. World Health Organization. Available from: https:// apps.who.int/iris/handle/10665/332196 [Accessed 3 June 2020].

6. Xia Y, Jin R, Zhao J, Li W, Shen H. Risk of COVID-19 for patients with cancer. Lancet Oncol 2020;21:e180.
7. Liang W, Guan W, Chen R, et al. Cancer patients in SARSCoV-2 infection: a nationwide analysis in China. Lancet Oncol 2020;21:335-337.

8. Lordick F, Mariette C, Haustermans K, Obermannová R, Arnold D; ESMO Guidelines Committee. Oesophageal cancer: ESMO Clinical Practice Guidelines for diagnosis, treatment and followup. Ann Oncol 2016;27 (Suppl 5):v50-v57.

9. Dubecz A, Kern M, Solymosi N, Schweigert M, Stein HJ. Predictors of lymph node metastasis in surgically resected T1 esophageal cancer. Ann Thorac Surg 2015;99:1879-1885.

10. Sgourakis G, Gockel I, Lang H. Endoscopic and surgical resection of T1a/T1b esophageal neoplasms: a systematic review. World $J$ Gastroenterol 2013;19:1424-1437.

11. Singhal S, Roy S. cT2N0 esophageal adenocarcinoma: predictors of lymph nodal involvement and clinical significance. J Thorac Dis 2019;11:S453-S456.

12. Mönig S, Chevallay M, Niclauss N, et al. Early esophageal cancer: the significance of surgery, endoscopy, and chemoradiation. Ann $N$ Y Acad Sci 2018;1434:115-123.

13. COVID-19 Clinical oncology frequently asked questions (FAQs). Available from: https://www.asco.org/asco-coronavirusinformation [Accessed 1 June 2020].

14. Clinical guide for the management of essential cancer surgery for adults during the coronavirus pandemic. Available from: https://www.uhb.nhs.uk/coronavirus-staff/downloads/pdf/ CoronavirusCancerManagement.pdf [Accessed 3 June 2020].

15. Mariette C, Dahan L, Mornex F, et al. Surgery alone versus chemoradiotherapy followed by surgery for stage I and II esophageal cancer: final analysis of randomized controlled phase III trial FFCD 9901. J Clin Oncol 2014;32:2416-2422.

16. Kitagawa Y, Uno T, Oyama T, et al. Esophageal cancer practice 
guidelines 2017 edited by the Japan Esophageal Society: part 1. Esophagus 2019;16:1-24.

17. Evans JA, Early DS, Chandraskhara V, et al; American Society for Gastrointestinal Endoscopy. The role of endoscopy in the assessment and treatment of esophageal cancer. Gastrointest Endosc 2013;77:328-334.

18. Li Y, Qin JJ, Wang Z, et al. Surgical treatment for esophageal cancer during the outbreak of COVID-19. Zhonghua Zhong Liu Za Zhi 2020;42:296-300.

19. Jones CM, Hawkins M, Mukherjee S, Radhakrishna G, Crosby T. Considerations for the treatment of oesophageal cancer with radiotherapy during the COVID-19 pandemic. Clin Oncol ( $R$ Coll Radiol) 2020;32:354-357.

20. Simcock R, Thomas TV, Estes C, et al. COVID-19: Global radiation oncology's targeted response for pandemic preparedness. Clin Transl Radiat Oncol 2020;22:55-68.

21. Smith JJ, Strombom P, Chow OS, et al. Assessment of a watch-andwait strategy for rectal cancer in patients with a complete response after neoadjuvant therapy. JAMA Oncol 2019;5:e185896.

22. Noordman BJ, Wijnhoven BPL, Lagarde SM, et al. Active surveillance in clinically complete responders after neoadjuvant chemoradiotherapy for esophageal or junctional cancer. Dis Esophagus 2017;30:1-8.

23. van der Wilk BJ, Noordman BJ, Neijenhuis LKA, et al. Active surveillance versus immediate surgery in clinically complete responders after neoadjuvant chemoradiotherapy for esophageal cancer: a multicenter propensity matched study. Ann Surg 2019 Oct 4 [Epub ahead of print]. doi: 10.1097/SLA.0000000000003636

24. Shapiro J, van Hagen P, Lingsma HF, et al; CROSS Study Group. Prolonged time to surgery after neoadjuvant chemoradiotherapy increases histopathological response without affecting survival in patients with esophageal or junctional cancer. Ann Surg 2014;260:807-813; discussion 813-814.

25. Noordman BJ, Spaander MCW, Valkema R, et al; SANO study group. Detection of residual disease after neoadjuvant chemoradiotherapy for oesophageal cancer (preSANO): a prospective multicentre, diagnostic cohort study. Lancet Oncol 2018;19:965-974.

26. Noordman BJ, Shapiro J, Spaander MC, et al; SANO study group. Accuracy of detecting residual disease after cross neoadjuvant chemoradiotherapy for esophageal cancer (preSANO Trial): rationale and protocol. JMIR Res Protoc 2015;4:e79.

27. Noordman BJ, Wijnhoven BPL, Lagarde SM, et al; SANO-study group. Neoadjuvant chemoradiotherapy plus surgery versus active surveillance for oesophageal cancer: a stepped-wedge cluster randomised trial. BMC Cancer 2018;18:142.

28. Comparison of systematic surgery versus surveillance and rescue surgery in operable oesophageal cancer with a complete clinical response to radiochemotherapy - full text view. Available from: https://clinicaltrials.gov/ct2/show/NCT02551458 [Accessed 1 June 2020].

29. Zhang X, Eyck BM, Yang Y, et al. Accuracy of detecting residual disease after neoadjuvant chemoradiotherapy for esophageal squamous cell carcinoma (preSINO trial): a prospective multicenter diagnostic cohort study. BMC Cancer 2020;20:194.

30. Triantafyllou T, Olson MT, Theodorou D, Schizas D, Singhal S. Enhanced recovery pathways vs standard care pathways in esophageal cancer surgery: systematic review and meta-analysis. Esophagus 2020;17:100-112.

31. Ueda M, Martins R, Hendrie PC, et al. Managing cancer care during the COVID-19 pandemic: agility and collaboration toward a common Goal. J Natl Compr Canc Netw 2020 Mar 20 [Epub ahead of print]. doi: 10.6004/jnccn.2020.7560

32. Francesco C, Pettke A, Michele B, Fabio P, Helleday T. Managing COVID-19 in the oncology clinic and avoiding the distraction effect. Ann Oncol 2020;31:553-555.

33. Filippi AR, Russi E, Magrini SM, Corvò R. Letter from Italy: first practical indications for radiation therapy departments during COVID-19 outbreak. Int J Radiat Oncol Biol Phys 2020 Mar 19 [Epub ahead of print]. doi: 10.1016/j.ijrobp.2020.03.007 